\title{
Predictive values of stool-based tests for mucosal healing among Taiwanese patients with ulcerative colitis: a retrospective cohort analysis
}

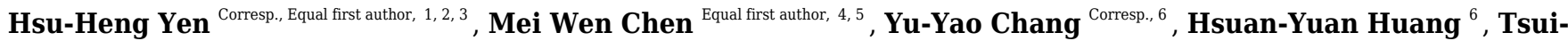 \\ Chun Hsu ${ }^{1}$, Yang-Yuan Chen ${ }^{1}$ \\ ${ }^{1}$ Division of Gastroenterology, Department of Internal Medicine, Changhua christian Hospital, Changhua, Taiwan \\ 2 Institute of Medicine, Chung Shan Medical and Dental College, Taichung, Taiwan \\ 3 General Education Center, Chienkuo Technology University, Changhua, Taiwan \\ 4 Department of Tumor Center, Changhua Christian Hospital, Changhua, Taiwan \\ 5 Information Management, Chien-Kuo Technology University, Changhua, Taiwan \\ 6 Department of Colorectal Surgery, Changhua christian Hospital, Changhua, Taiwan \\ Corresponding Authors: Hsu-Heng Yen, Yu-Yao Chang \\ Email address: 91646@cch.org.tw, 177176@cch.org.tw
}

Background/Purpose. Over the past two decades, ulcerative colitis (UC) has emerged in the Asia Pacific area, and its treatment goal has shifted from symptom relief to endoscopic remission. Endoscopy is the gold standard for the assessment of mucosal healing; however, it is an invasive method. Fecal calprotectin (FC) is a non-invasive stool-based inflammatory marker, which has been used to monitor mucosal healing status, but it is expensive. By contrast, the immune fecal occult blood test (iFOBT) is a widely utilized stool-based screening tool for colorectal cancer. In this study, we compared the predictive values of iFOBT and FC for mucosal healing in Taiwanese patients with UC. Methods. A total of 50 patients with UC identified via the electronic clinical database of Changhua Christian Hospital, Taiwan, were retrospectively enrolled from January 2018 to July 2019. Results of iFOBT, FC level, and blood tests as well as Mayo scores were reviewed and analyzed. Colonic mucosa was evaluated using the endoscopic Mayo subscore. Results. The average age of the patients was 46 years, and $62 \%$ of the patients were men. Disease distribution was as follows: E1 (26\%), E2 (40\%), and E3 (34\%). Complete mucosal healing (Mayo score $=0$ ) was observed in $30 \%$ of patients. Endoscopic mucosal healing with a Mayo score of 0 or 1 was observed in $62 \%$ of the patients. Results of FC and iFOBT were compared among patients with and without mucosal healing. Predictive cutoff values were analyzed using receiver operating characteristics curves. iFOBT and FC had similar area under the curve for both complete mucosal healing $(0.813$ vs. 0.769 , respectively, $p=$ 0.5581 ) and endoscopic mucosal healing (0.906 vs. 0.812 , respectively, $p=0.1207)$.

Conclusion. In daily clinical practice, FC and iFOBT do not differ in terms of predictive 
values for mucosal healing among Taiwanese patients with UC. 
1 Predictive values of stool-based tests for mucosal healing among Taiwanese patients with

2 ulcerative colitis: A Retrospective Cohort Analysis

3

4 Hsu-Heng Yen ${ }^{1,2,3}$, May-Wen Chen ${ }^{4,5}$, Yu-Yao Chang ${ }^{6}$, Hsuan-Yuan Huang ${ }^{6}$, Tsui-Chun Hsu ${ }^{1}$,

5 Ya-Huei Zeng ${ }^{1}$, Yang-Yuan Chen ${ }^{1}$

6

$7 \quad{ }^{1}$ Division of Gastroenterology, Department of Internal Medicine, Changhua Christian Hospital,

8 Changhua, Taiwan

9 2Institute of Medicine, Chung Shan Medical and Dental College, Taichung, Taiwan

$10{ }^{3}$ General Education Center, Chienkuo Technology University, Changhua, Taiwan

$11{ }^{4}$ Department of Tumor Center, Changua Christian Hospital, ChangHua, Taiwan

$12{ }^{5}$ Department of Information Management, Chien-Kuo Technology University, ChungHua,

13 Taiwan

$14{ }^{6}$ Department of Colorectal Surgery, Changhua Christian Hospital, Changhua, Taiwan

15

16 Corresponding and reprint requests to:

17 Hsu-Heng Yen

18 E-mail: 91646@cch.org.tw, blaneyen@gmail.com

Peer] reviewing PDF | (2020:05:48530:1:1:NEW 18 Jun 2020) 
19 Division of Gastroenterology, Department of Internal Medicine, Changhua Christian Hospital,

20 Changhua, Taiwan

21 


\section{Abstract}

24 Background/Purpose. Over the past two decades, ulcerative colitis (UC) has emerged in the

25 Asia Pacific area, and its treatment goal has shifted from symptom relief to endoscopic

26 remission. Endoscopy is the gold standard for the assessment of mucosal healing; however, it is

27 an invasive method. Fecal calprotectin (FC) is a non-invasive stool-based inflammatory marker,

28 which has been used to monitor mucosal healing status, but it is expensive. By contrast, the

29 immune fecal occult blood test (iFOBT) is a widely utilized stool-based screening tool for

30 colorectal cancer. In this study, we compared the predictive values of iFOBT and FC for mucosal

31 healing in Taiwanese patients with UC.

32 Methods. A total of 50 patients with UC identified via the electronic clinical database of

33 Changhua Christian Hospital, Taiwan, were retrospectively enrolled from January 2018 to July

34 2019. Results of iFOBT, FC level, and blood tests as well as Mayo scores were reviewed and

35 analyzed. Colonic mucosa was evaluated using the endoscopic Mayo subscore.

36 Results. The average age of the patients was 46 years, and $62 \%$ of the patients were men.

37 Disease distribution was as follows: E1 (26\%), E2 (40\%), and E3 (34\%). Complete mucosal

38 healing (Mayo score $=0$ ) was observed in 30\% of patients. Endoscopic mucosal healing with a

39 Mayo score of 0 or 1 was observed in $62 \%$ of the patients. Results of FC and iFOBT were 
40 compared among patients with and without mucosal healing. Predictive cutoff values were

41 analyzed using receiver operating characteristics curves. iFOBT and FC had similar area under

42 the curve for both complete mucosal healing $(0.813$ vs. 0.769 , respectively, $\mathrm{p}=0.5581)$ and

43 endoscopic mucosal healing (0.906 vs. 0.812, respectively, $\mathrm{p}=0.1207)$.

44 Conclusion. In daily clinical practice, FC and iFOBT do not differ in terms of predictive values

45 for mucosal healing among Taiwanese patients with UC. 


\section{Introduction}

48

49 Ulcerative colitis (UC) is a chronic inflammatory disease of the colon (Wei et al. 2017; Yen et al.

50 2017; Yen et al. 2019); without adequate treatment and monitoring of the disease, it may lead to

51 complications such as bleeding, perforation, and development of malignancy (Wei et al. 2017).

52 The state-of-the-art treatment goal for UC has shifted from clinical remission with symptom

53 control to endoscopic remission using the treat-to-target strategy (Jackson \& De Cruz 2019;

54 Rubin et al. 2019; Wei et al. 2017). Fecal calprotectin (FC) is a non-invasive fecal marker

55 commonly used in Western countries to determine mucosal healing (Freeman et al. 2019;

56 Motaganahalli et al. 2019); however, FC is expensive compared with the immune fecal occult

57 blood test (iFOBT) and is not reimbursed for clinical use in Taiwan. By contrast, colonoscopy

58 remains the gold standard for the assessment of colonic mucosal status, enables screening for

59 colitis-associated malignancies (Wei et al. 2017; Yen et al. 2017), and costs less in Asia than in

60 Western countries (Chang et al. 2020; Yen \& Hsu 2019). However, colonoscopy is invasive and

61 patients may show slight reluctance to undergo this procedure (Lin et al. 2015; Yen \& Hsu

62 2019). Moreover, colonoscopy may induce worsening of UC or result in complications (Wei et

63 al. 2017).

64 
65 Quantitative iFOBT has replaced guaiac-based measures of stool hemoglobin concentrations

66 owing to its better performance in colorectal cancer screening. Recent studies conducted in

67 Canada (Ma et al. 2017), Japan (Nakarai et al. 2013), Korea (Ryu et al. 2016), and China (Shi et

68 al. 2017) used iFOBT to predict mucosal healing among patients with UC (Dai et al. 2018).

69 Meanwhile, only limited data are available on the comparison of the relative predictive values of

70 iFOBT and FC for mucosal healing (Kim et al. 2020; Naganuma et al. 2020; Ryu et al. 2019;

71 Takashima et al. 2015). In 2018, with the aim of improving clinical practice, we began

72 performing $\mathrm{iFOBT}$ and $\mathrm{FC}$ at our institution for disease monitoring of patients with

73 inflammatory bowel disease (IBD). In the present study, we aimed to compare the predictive

74 values of iFOBT and FC for mucosal healing in Taiwanese patients with UC.

75 


\section{Materials \& Methods}

77

78 The medical records of patients diagnosed with UC between January 2018 and July 2019 at

79 Changhua Christian Hospital, Taiwan, were retrospectively reviewed. From January 2018,

80 patients diagnosed with IBD, including UC and Crohn's disease, received integrated hospital

81 care by a trained IBD nurse, who recorded clinical symptoms using the Mayo scoring system for

82 UC severity during each outpatient clinic visit. Patients with UC underwent screening

83 colonoscopy for the evaluation of disease activity and monitoring of malignancy. Laboratory

84 tests were conducted annually during the follow-up period. Starting from 2018, prior to

85 colonoscopy, stool samples were collected for an immunochemical occult test to evaluate and

86 compare the usefulness of iFOBT and FC for evaluating IBD activity in clinical practice.

87 Samples for iFOBT were analyzed using the HM-JACK system (Kyowa Medex, Shizuoka,

88 Japan), which is a fully automated quantitative iFOBT system. The HM-JACK system can

89 accurately measure fecal hemoglobin concentrations within a range of $7-400 \mathrm{ng} / \mathrm{mL}$. Samples for

90 FC were analyzed using the commercially available Quantum Blue ${ }^{\circledR}$ fCAL test (Buhlmann

91 Laboratories AG, Schonenbuch, Switzerland).

92

93 Patients enrolled in the present study met the following inclusion criteria: (a) diagnosed with UC 
94 for >6 months; (b) tested by both iFOBT and FC within 1 month prior to colonoscopy; (c) had

95 medical records of clinical symptoms, partial Mayo score, and laboratory test results, and (d) had

96 undergone colonoscopy with documentation of the endoscopic Mayo scoring system. The Mayo

97 score and laboratory test data were obtained 1 month before colonoscopy.

99 The requirement for informed consent for data extraction was waived by the institutional review

100 board because of the retrospective design of the study and the minimal risk involved. Mayo

101 scores, including stool frequency, rectal bleeding, endoscopy findings, and overall clinical

102 evaluation, were used to evaluate UC severity using 0-3 points for each component. The partial

103 Mayo score comprised three non-endoscopic variables. An endoscopic Mayo score of 0 indicates

104 complete mucosal healing $(\mathrm{CMH})$, and an endoscopic Mayo score of 0 or 1 on colonoscopy

105 indicates endoscopic mucosal healing (EMH). The primary endpoint of this study was to

106 compare the predictive values of stool-based tests for the mucosal status, i.e., $\mathrm{CMH}$ and EMH.

107 The secondary endpoint was to compare the correlation between endoscopy activity and stool-

108 based tests and blood-based systematic inflammatory markers. The study complied with the

109 World Medical Association Declaration of Helsinki for medical research involving human

110 subjects including research on identifiable human material and data. The study was approved by

111 the Institutional Review Board of Changhua Christian Hospital (approval number: CCH IRB 
112 190814).

113

114 Statistical analysis

115 The extracted data were organized using Microsoft Excel and analyzed using MedCalc Statistical

116 Software version 19.16 (MedCalc Software bvba, Ostend, Belgium; https://www.medcalc.org;

117 2020). Continuous data are expressed as means and standard deviations or as medians and

118 interquartile ranges for normally and non-normally distributed data, respectively. Categorical

119 variables are presented as numbers and percentages. The mean values of normally distributed

120 variables were compared by an independent sample's Student's $t$-test. Mann-Whitney $U$-test and

121 Kruskal-Wallis test were performed to compare the mean values of 2 and $\geq 3$ groups of variables,

122 respectively, with non-normal distributions. The frequencies of categorical variables were

123 compared using Pearson's $\chi^{2}$ or Fisher's exact test, as appropriate. Spearman's rank correlation

124 was performed to determine the correlation between fecal test data and UC severity as reflected

125 by the Mayo score. Receiver operating characteristic (ROC) curve analysis was conducted to

126 determine the best cutoff values of iFOBT and FC levels for predicting mucosal healing. All $P$ -

127 values of $<0.05$ were considered statistically significant. 


\section{Results}

130

131 Clinical features of patients with UC

132 During the study period, a total of 102 patients with UC received treatment at the hospital and 50

133 met the inclusion criteria. The clinical characteristics of all patients are presented in Table 1 . The

134 average age of the patients was 46 years, and the majority of them were men (31 men, 19

135 women). The median duration of UC was 3.5 years. Based on the Montreal classification, the

136 diseases observed included proctitis (E1, 26\%), left-sided UC (E2, 40\%), and extensive UC (E3,

137 34\%). Further, there were 8\% current smokers, 24\% ever-smokers, and 68\% non-smokers. Oral

138 5-aminosalicylic acid (5-ASA) was administered to $94 \%$ of the patients, followed by rectal 5-

139 ASA (54\%), steroids (34\%), and immune modulators (24\%). CMH with an endoscopic Mayo

140 score of 0 was achieved in $30 \%$ of the patients, whereas EMH)with an endoscopic Mayo score of

$141 \leq 1$ was achieved in $62 \%$.

142

143 Comparison of patients with and without mucosal healing

144 In our cohort, $30 \%(15 / 50)$ of the patients exhibited CMH as evaluated by colonoscopy; the

145 remaining patients had endoscopic Mayo scores of $1(33.3 \%), 2(18.52 \%)$, and $3(22.22 \%)$

146 (Table 2). Age, sex, disease distribution, and drugs used did not differ between patients with 
$147 \mathrm{CMH}$ and those without $\mathrm{CMH}$. Compared with patients without $\mathrm{CMH}$, those with $\mathrm{CMH}$ had

148 lower $\operatorname{iFOBT}(P=0.003)$ and $\mathrm{FC}(P=0.0028)$ values.

$149 \mathrm{EMH}$, as indicated by an endoscopic Mayo score of 0 or 1 on colonoscopy, was achieved in $65 \%$

150 (Table 3). Age, sex, disease distribution, and drugs used did not differ between those with EMH

151 and those without EMH. Compared with patients without EMH, those with EMH had lower

152 iFOBT $(P<0.001)$ and FC $(P=0.0002)$ values.

153

154 Relative predictive values of $i F O B T$ and FC for mucosal healing

155 Both iFOBT and FC showed moderate correlations with the total endoscopic Mayo score (Fig.

156 1). The correlation among stool-based tests (FC and iFOBT), blood inflammatory markers (CRP

157 and ESR), and endoscopically evaluated UC activity was analyzed using Spearman's rank

158 correlation coefficient (Table 4). Compared with FC, ESR, and CRP, iFOBT exhibited a higher

159 correlation with the endoscopic Mayo score. As illustrated in Fig. 2, an iFOBT criterion of $\leq 30$

$160 \mathrm{ng} / \mathrm{mL}$ had $93.33 \%$ sensitivity and $71.43 \%$ specificity in terms of predicting $\mathrm{CMH}$, whereas an

$161 \mathrm{iFOBT}$ criterion of $\leq 43 \mathrm{ng} / \mathrm{mL}$ had $80.65 \%$ sensitivity and $100 \%$ specificity in predicting EMH.

162 As illustrated in Fig. 3, an FC criterion of $\leq 156 \mu \mathrm{g} / \mathrm{g}$ had $86.67 \%$ sensitivity and $62.86 \%$

163 specificity in terms of predicting $\mathrm{CMH}$, whereas an $\mathrm{FC}$ criterion of $\leq 156 \mu \mathrm{g} / \mathrm{g}$ had $74.19 \%$

164 sensitivity and 84.21\% specificity in predicting EMH. As illustrated in Fig. 4, the ROC curve 
165 analysis of the ability to predict mucosal healing showed that iFOBT results tended to have 166 higher AUCs for both $\mathrm{CMH}$ and $\mathrm{EMH}$ than FC values.

167

168 


\section{Discussion}

170

171 In the present study, we compared the predictive values of iFOBT and FC for mucosal healing

172 among Taiwanese patients with UC in clinical practice. Our findings were consistent with those

173 of previous studies, which demonstrated that iFOBT and FC had similar predictive values for

174 predicting EMH among patients with UC (Kim et al. 2018; Kim et al. 2020; Ma et al. 2017;

175 Naganuma et al. 2020; Ryu et al. 2019; Takashima et al. 2015). iFOBT might be used as an

176 alternative non-invasive monitoring tool for patients with UC, particularly in Taiwan, where

177 other fecal tests such as FC level are not widely available and are more expensive.

178

179 The incidence of IBD is increasing worldwide, particularly in the Asia Pacific region (Jung

180 2020; Wei et al. 2013; Yen et al. 2019). Our recent national cohort study from Taiwan (Yen et al.

181 2019) reported a six-fold increase to $12.8 / 100,000$ in the prevalence of UC over the past 15

182 years. The treatment goal for IBD has shifted from clinical remission to biochemical remission,

183 endoscopic remission, and histological healing (Rubin et al. 2019; Wei et al. 2017). Patients with

184 UC are typically evaluated using clinical symptoms based on the Mayo scoring system.

185 However, subjective reports of symptoms such as bowel frequency may not correlate well with

186 the endoscopy findings. Uncontrolled but asymptomatic inflammation may increase the risk of 
187 disease relapse or subsequent development of complications(Peyrin-Biroulet et al. 2015).

188 Colonoscopy has been the gold standard for the evaluation of mucosal status; however, it is

189 invasive and less acceptable by patients compared with non-invasive tests such as blood and

190 stool tests. In the present study, $25 \%$ of the patients with an endoscopic Mayo score of 3 for

191 severe colonic inflammation reported no rectal bleeding and/or had normal or near-normal stool

192 frequencies. Therefore, relying on patient-reported symptoms may underestimate the severity of

193 colonic inflammation.

194 Calprotectin is a calcium-binding protein, which is mainly found in neutrophils (Wei 2016). FC

195 has been correlated with mucosal inflammation and has been used as a surrogate biomarker for

196 evaluating IBD activity in Western countries (Kim et al. 2020; Lin et al. 2015; Takashima et al.

197 2015). Although non-invasive, FC is not readily available in Asia and it costs the same as or

198 more than colonoscopy in Taiwan (Lin et al. 2015; Wei et al. 2017); therefore, its use in our

199 clinical practice has been limited. By contrast, quantitative iFOBT is a stool-based test for colon

200 cancer screening and is available worldwide (Yen \& Hsu 2019). Unlike Crohn's disease, UC

201 involves the superficial colonic wall; therefore, mucosal hemorrhage may be used as a surrogate

202 marker for predicting mucosal damage (Kuriyama et al. 2010).

204 In a study from Canada, Ma et al. (Ma et al. 2017) reported similar predictive values of iFOBT 
205 and FC for mucosal healing in patients with IBD, particularly with UC. Further, in Asia, the

206 interest in the use of iFOBT as a test for predicting mucosal damage among patients with UC has

207 been increasing (Naganuma et al. 2019; Nakarai et al. 2013; Ryu et al. 2016; Ryu et al. 2019; Shi

208 et al. 2017; Takashima et al. 2015). Using different cutoff levels and test kits, iFOBT was

209 reported to predict endoscopy mucosal healing with 58\%-94.9\% sensitivity and 38.3\%-100\%

210 specificity. Data on the optimal cutoff levels of these stool-based tests for predicting EMH has

211 varied among different studies due to the different testing kits used.

212

213 Although the UC treatment guidelines in Taiwan have recommended FC as a biomarker for

214 detecting colonic inflammation and for evaluating disease activity (Wei et al. 2017), this test is

215 not widely available and is not reimbursed by the national insurance in Taiwan. Few studies have

216 compared the performances of iFOBT and FC (Table 5), and the results are conflicting.

217 Takashima et al. (Takashima et al. 2015) conducted the first study on the use of FC and iFOBT

218 to predict the mucosal status of 92 patients with UC. They found that both iFOBT and FC

219 efficiently predicted mucosal healing in UC, but compared with FC, iFOBT appeared to be more

220 sensitive in predicting CMH. Ryu et al. (Ryu et al. 2019) from Korea found a more accurate

221 prediction of endoscopic activity with FC than with iFOBT. Kim et al. (Kim et al. 2018), also

222 from Korea, found that compared with iFOBT, FC was better in predicting CMH but with a 
223 similar performance with regard to predicting EMH (Kim et al. 2018; Kim et al. 2020). To the

224 best of our knowledge, the present study is the first from Taiwan to compare the relative

225 predictive values of iFOBT and FC. Our findings are consistent with those of a recent Japanese

226 nationwide cohort study (Naganuma et al. 2020), which suggested similar predictive values of

227 iFOBT and FC for the mucosal status of patients with UC. Given the low cost and similar

228 predictive value of FC, iFOBT may be advantageous as a first-line monitoring tool for patients

229 with UC in Taiwan.

230

231 There are several limitations to this study. First, our study had a single-center retrospective

232 design; therefore, the results require validation by further large-scale studies outside Taiwan.

233 Endoscopic findings were retrospectively reviewed and may have been biased due to the fact that

234 different endoscopists were involved. Second, we only performed one iFOBT and FC test before

235 colonoscopy owing to the high cost of FC. Therefore, we were not able to investigate and

236 compare the potential diagnostic relevance of iFOBT and FC in predicting treatment response or

237 disease relapse after remission. The combined use of both fecal tests is promising, and Japanese

238 researchers have shown promising results with regard to predicting disease relapse (Naganuma et

239 al. 2020; Nakarai et al. 2018). Third, colonic polyps or cancer was not identified in any of our

240 patients during colonoscopy. Theoretically, iFOBT cannot differentiate between UC and 
241 colorectal neoplasms. Importantly, iFOBT should not replace surveillance colonoscopy; we

242 suggest its use as a monitoring tool to assess disease activity rather than for cancer surveillance.

243

244 Conclusions

245

246 The increased prevalence of UC in Taiwan has raised the need for a practical tool to monitor

247 disease activity. Our study found similar predictive values of $\mathrm{FFOBT}$ and FC for both $\mathrm{CMH}$ and

248 EMH. Therefore, iFOBT might be useful as a first-line non-invasive tool in clinical practice to

249 evaluate disease severity and may assist in clinical decision making.

250

251 Acknowledgments

252

253 The authors received funding for this study from Changhua Christian Hospital (106-IRP-CCH-

254030 and 108-CCHIRP-018).

255

256 References

257

258

Chang CW, Tu CH, Chou JW, Huang TY, Hsu WH, Wang YP, Chen CC, Chung CS, Lin CP, Lin WC,

Tai CM, Wang HY, and Chen MJ. 2020. Endoscopic management of strictures in patients 
260

261

262

263

264

265

266

267

268

269

270

271

272

273

274

275

276

277

278

279

280

281

282

283

284

285

286

287

288

289

290

291

292

293

294

295

with Crohn's disease - A multi-center experience in Taiwan. J Formos Med Assoc. 10.1016/j.jfma.2019.12.005

Dai C, Jiang M, Sun MJ, and Cao Q. 2018. Fecal immunochemical test for predicting mucosal healing in ulcerative colitis patients: A systematic review and meta-analysis. $J$ Gastroenterol Hepatol 33:990-997. 10.1111/jgh.14121

Freeman K, Willis BH, Fraser H, Taylor-Phillips S, and Clarke A. 2019. Faecal calprotectin to detect inflammatory bowel disease: a systematic review and exploratory meta-analysis of test accuracy. BMJ Open 9:e027428. 10.1136/bmjopen-2018-027428

Jackson BD, and De Cruz P. 2019. Quality of Care in Patients With Inflammatory Bowel Disease. Inflamm Bowel Dis 25:479-489. 10.1093/ibd/izy276

Jung YS. 2020. Trends in Healthcare Costs for Inflammatory Bowel Disease in South Korea. Gut Liver 14:3-4. 10.5009/gnl19374

Kim DJ, Jeoun YM, Lee DW, Koo JS, and Lee SW. 2018. Usefulness of fecal immunochemical test and fecal calprotectin for detection of active ulcerative colitis. Intest Res 16:563-570. 10.5217/ir.2018.00020

Kim ES, Lee HS, Kim SK, Kim EY, Jang BI, Kim KO, Yang CH, Lee YJ, Crohn's, and Colitis Association in D-G. 2020. Fecal calprotectin is more accurate than fecal immunochemical test for predicting mucosal healing in quiescent ulcerative colitis: a prospective multicenter study. Scand J Gastroenterol:1-6. 10.1080/00365521.2020.1714716

Kuriyama M, Kato J, Takemoto K, Hiraoka S, Okada H, and Yamamoto K. 2010. Prediction of flare-ups of ulcerative colitis using quantitative immunochemical fecal occult blood test. World J Gastroenterol 16:1110-1114. 10.3748/wjg.v16.i9.1110

Lin WC, Wong JM, Tung CC, Lin CP, Chou JW, Wang HY, Shieh MJ, Chang CH, Liu HH, Wei SC, and Taiwan Society of Inflammatory Bowel Disease Multicenter S. 2015. Fecal calprotectin correlated with endoscopic remission for Asian inflammatory bowel disease patients. World J Gastroenterol 21:13566-13573. 10.3748/wjg.v21.i48.13566

Ma C, Lumb R, Walker EV, Foshaug RR, Dang TT, Verma S, Huang VW, Kroeker KI, Wong K, Dieleman LA, Fedorak RN, and Halloran BP. 2017. Noninvasive Fecal Immunochemical Testing and Fecal Calprotectin Predict Mucosal Healing in Inflammatory Bowel Disease: A Prospective Cohort Study. Inflamm Bowel Dis 23:1643-1649. 10.1097/MIB.0000000000001173

Motaganahalli S, Beswick L, Con D, and van Langenberg DR. 2019. Faecal calprotectin delivers on convenience, cost reduction and clinical decision-making in inflammatory bowel disease: a real-world cohort study. Intern Med J 49:94-100. 10.1111/imj.14027

Naganuma M, Kobayashi T, Nasuno M, Motoya S, Kato S, Matsuoka K, Hokari R, Watanabe C, Sakamoto H, Yamamoto H, Sasaki M, Watanabe K, lijima H, Endo Y, Ichikawa H, Ozeki K, 
296

297

298

299

300

301

302

303

304

305

306

307

308

309

310

311

312

313

314

315

316

317

318

319

320

321

322

323

324

325

326

327

328

329

330

331

Tanida S, Ueno N, Fujiya M, Sako M, Takeuchi K, Sugimoto S, Abe T, Hibi T, Suzuki Y, and Kanai T. 2019. Significance of Conducting 2 Types of Fecal Tests in Patients with Ulcerative Colitis. Clin Gastroenterol Hepatol. 10.1016/j.cgh.2019.07.054

Naganuma M, Kobayashi T, Nasuno M, Motoya S, Kato S, Matsuoka K, Hokari R, Watanabe C, Sakamoto H, Yamamoto H, Sasaki M, Watanabe K, lijima H, Endo Y, Ichikawa H, Ozeki K, Tanida S, Ueno N, Fujiya M, Sako M, Takeuchi K, Sugimoto S, Abe T, Hibi T, Suzuki Y, and Kanai T. 2020. Significance of Conducting 2 Types of Fecal Tests in Patients With Ulcerative Colitis. Clin Gastroenterol Hepatol 18:1102-1111.e1105. 10.1016/j.cgh.2019.07.054

Nakarai A, Hiraoka S, Takahashi S, Inaba T, Higashi R, Mizuno M, Takashima S, Inokuchi T, Sugihara Y, Takahara M, Harada K, Kato J, and Okada H. 2018. Simultaneous Measurements of Faecal Calprotectin and the Faecal Immunochemical Test in Quiescent Ulcerative Colitis Patients Can Stratify Risk of Relapse. J Crohns Colitis 12:71-76. 10.1093/ecco-jcc/jjx118

Nakarai A, Kato J, Hiraoka S, Kuriyama M, Akita M, Hirakawa T, Okada H, and Yamamoto K. 2013. Evaluation of mucosal healing of ulcerative colitis by a quantitative fecal immunochemical test. Am J Gastroenterol 108:83-89. 10.1038/ajg.2012.315

Peyrin-Biroulet L, Sandborn W, Sands BE, Reinisch W, Bemelman W, Bryant RV, D'Haens G, Dotan I, Dubinsky M, Feagan B, Fiorino G, Gearry R, Krishnareddy S, Lakatos PL, Loftus EV, Jr., Marteau P, Munkholm P, Murdoch TB, Ordas I, Panaccione R, Riddell RH, Ruel J, Rubin DT, Samaan M, Siegel CA, Silverberg MS, Stoker J, Schreiber S, Travis S, Van Assche G, Danese S, Panes J, Bouguen G, O'Donnell S, Pariente B, Winer S, Hanauer S, and Colombel JF. 2015. Selecting Therapeutic Targets in Inflammatory Bowel Disease (STRIDE): Determining Therapeutic Goals for Treat-to-Target. Am J Gastroenterol 110:1324-1338. 10.1038/ajg.2015.233

Rubin DT, Ananthakrishnan AN, Siegel CA, Sauer BG, and Long MD. 2019. ACG Clinical Guideline: Ulcerative Colitis in Adults. Am J Gastroenterol 114:384-413. 10.14309/ajg.0000000000000152

Ryu DG, Kim HW, Park SB, Kang DH, Choi CW, Kim SJ, and Nam HS. 2016. Assessment of disease activity by fecal immunochemical test in ulcerative colitis. World J Gastroenterol 22:10617-10624. 10.3748/wjg.v22.i48.10617

Ryu DG, Kim HW, Park SB, Kang DH, Choi CW, Kim SJ, and Nam HS. 2019. Clinical implications of fecal calprotectin and fecal immunochemical test on mucosal status in patients with ulcerative colitis. Medicine (Baltimore) 98:e17080. 10.1097/MD.0000000000017080

Shi HY, Chan FKL, Chan AWH, Higashimori A, Kyaw M, Ching JYL, Luk AKC, Wong SH, Wu JCY, Sung JJY, and Ng SC. 2017. Accuracy of Faecal Immunochemical Test to Predict 
332

333

334

335

336

337

338

339

340

341

342

343

344

345

346

347

348

349

350

351

352

353

354

355

356

357

358

359
Endoscopic and Histological Healing in Ulcerative Colitis: A Prospective Study Based on Validated Histological Scores. J Crohns Colitis 11:1071-1077. 10.1093/ecco-jcc/jjx088

Takashima S, Kato J, Hiraoka S, Nakarai A, Takei D, Inokuchi T, Sugihara Y, Takahara M, Harada K, Okada H, Tanaka T, and Yamamoto K. 2015. Evaluation of Mucosal Healing in Ulcerative Colitis by Fecal Calprotectin Vs. Fecal Immunochemical Test. Am J Gastroenterol 110:873-880. 10.1038/ajg.2015.66

Wei SC. 2016. Could fecal calprotectin enter mainstream use for diagnosing and monitoring inflammatory bowel disease? Intest Res 14:293-294. 10.5217/ir.2016.14.4.293

Wei SC, Chang TA, Chao TH, Chen JS, Chou JW, Chou YH, Chuang CH, Hsu WH, Huang TY, Hsu TC, Lin CC, Lin HH, Lin JK, Lin WC, Ni YH, Shieh MJ, Shih IL, Shun CT, Tsang YM, Wang CY, Wang HY, Weng MT, Wu DC, Wu WC, Yen HH, and Wong JM. 2017. Management of ulcerative colitis in Taiwan: consensus guideline of the Taiwan Society of Inflammatory Bowel Disease. Intest Res 15:266-284. 10.5217/ir.2017.15.3.266

Wei SC, Lin MH, Tung CC, Weng MT, Kuo JS, Shieh MJ, Wang CY, Ho WC, Wong JM, and Chen PC. 2013. A nationwide population-based study of the inflammatory bowel diseases between 1998 and 2008 in Taiwan. BMC Gastroenterol 13:166. 10.1186/1471-230X-13166

Yen HH, Chang CW, Chou JW, and Wei SC. 2017. Balloon-Assisted Enteroscopy and Capsule Endoscopy in Suspected Small Bowel Crohn's Disease. Clin Endosc 50:417-423. 10.5946/ce.2017.142

Yen $\mathrm{HH}$, and Hsu YC. 2019. Changing from two- to one-operator colonoscopy insertion technique is feasible with similar quality outcomes. JGH Open 3:159-162. 10.1002/jgh3.12124

Yen HH, Weng MT, Tung CC, Wang YT, Chang YT, Chang CH, Shieh MJ, Wong JM, and Wei SC. 2019. Epidemiological trend in inflammatory bowel disease in Taiwan from 2001 to 2015: a nationwide populationbased study. Intest Res 17:54-62. 10.5217/ir.2018.00096 
Table $\mathbf{1}$ (on next page)

Demographic data of the patients 
1 Table 1. Demographic data of the patients

\begin{tabular}{|c|c|}
\hline Clinical variable & \\
\hline $\operatorname{Sex}(M / F)$ & $31 / 19$ \\
\hline Age, years (mean, 95\% CI) & $46(39 \sim 52.4)$ \\
\hline Disease duration (median, IQR) & $3.5(2-6)$ \\
\hline Disease distribution (E1/E2/E3) & $13 / 20 / 17(26 \% / 40 \% / 34 \%)$ \\
\hline $\begin{array}{l}\text { Smoking status (nonsmoker/current } \\
\text { smoker/ever-smoker) }\end{array}$ & $34 / 4 / 12(68 \% / 8 \% / 24 \%)$ \\
\hline Current medication & \\
\hline - $\quad$ steroid $(\mathrm{Y} / \mathrm{N})$ & $17 / 33(34 \% / 66 \%)$ \\
\hline - $\quad$ oral 5-ASA $(\mathrm{Y} / \mathrm{N})$ & $47 / 3(94 \% / 6 \%)$ \\
\hline - $\quad$ rectal 5-ASA $(\mathrm{Y} / \mathrm{N})$ & $27 / 23(54 \% / 47 \%)$ \\
\hline - $\quad$ immune modulator $(\mathrm{Y} / \mathrm{N})$ & $12 / 38(24 \% / 76 \%)$ \\
\hline - biologic agent $(\mathrm{Y} / \mathrm{N})$ & $1 / 49(2 \% / 98 \%)$ \\
\hline WBC count, $10^{3} / \mu \mathrm{L}$ (median, IQR) & $6.5(5.6-7.4)$ \\
\hline Hemoglobin level, g/dL (median, IQR) & $14.15(13.26-14.6)$ \\
\hline Platelet count, $10^{3} / \mu \mathrm{L}$ (median, IQR) & $261(229.83-295.95)$ \\
\hline NLR (median, IQR) & $2.26(1.84-2.74)$ \\
\hline CRP level, mg/dL (median, IQR) & $0.13(0.09-0.24)$ \\
\hline ESR, mm/h (median, IQR) & $10(8-13.8)$ \\
\hline Endoscopy Mayo score $(0 / 1 / 2 / 3)$ & $15 / 16 / 8 / 11(30 \% / 32 \% / 16 \% / 22 \%))$ \\
\hline iFOBT, ng/ml (median, IQR) & $44.5(7-101.7)$ \\
\hline FC, $\mu \mathrm{g} / \mathrm{g}$ (median,IQR) & $135.88(83.06-651.6)$ \\
\hline
\end{tabular}


2 5-ASA, 5-aminosalicylic acid; CRP, C-reactive protein; ESR, erythrocyte sedimentation rate;

3 iFOBT, immune fecal occult blood test; WBC, white blood cell; FC, fecal calprotectin 
Table 2 (on next page)

Comparison of patients with and without complete mucosal healing 
1 Table 2. Comparison of patients with and without complete mucosal healing

\begin{tabular}{|c|c|c|c|}
\hline & CMH $(+)(n=15)$ & CMH (-) $(\mathrm{n}=35)$ & $P$-value \\
\hline Age (mean, SD) & $48.13(15.17)$ & $45.71(12.51)$ & 0.560 \\
\hline WBC count & $5.7(5.4-8.6)$ & $6.7(5.4-8.2)$ & 0.8489 \\
\hline \multicolumn{4}{|l|}{ (median, IQR) } \\
\hline $\mathrm{Hb}$ (median, IQR) & $16.7(13.8-15.3)$ & $17(11.4-14.7)$ & 0.0284 \\
\hline Platelet (median, & $233(192-265)$ & $287(220-350))$ & 0.0466 \\
\hline \multicolumn{4}{|l|}{ IQR) } \\
\hline $\mathrm{iFOBT}, \mathrm{ng} / \mathrm{mL}$ & $7(7-22.5)$ & $121(8.5-400)$ & 0.0003 \\
\hline \multicolumn{4}{|l|}{ (median, IQR) } \\
\hline $\mathrm{FC}, \mu \mathrm{g} / \mathrm{g}$ (median, & $59.95(12.88-110.5)$ & $555(79.96-1687.34)$ & 0.0028 \\
\hline \multicolumn{4}{|l|}{ IQR) } \\
\hline Disease & $3 / 7 / 5$ & $10 / 13 / 12$ & 0.7643 \\
\hline \multicolumn{4}{|l|}{ distribution } \\
\hline \multicolumn{4}{|l|}{$(\mathrm{E} 1 / \mathrm{E} 2 / \mathrm{E} 3)$} \\
\hline Mayo score stool & $4 / 9 / 2 / 0$ & $7 / 13 / 10 / 5$ & 0.0694 \\
\hline \multicolumn{4}{|l|}{ frequency $(0 / 1 / 2 / 3)$} \\
\hline Mayo score rectal & $12 / 3 / 0 / 0$ & $17 / 8 / 8 / 2$ & 0.0162 \\
\hline \multicolumn{4}{|l|}{ bleeding $(0 / 1 / 2 / 3)$} \\
\hline Physician rating of & $9 / 6 / 0 / 0$ & $7 / 20 / 7 / 1$ & 0.0032 \\
\hline \multicolumn{4}{|l|}{ disease activity } \\
\hline$(0 / 1 / 2 / 3)$ & & & \\
\hline Current medication & & & \\
\hline
\end{tabular}




$\begin{array}{llcr}- \text { steroids }(\%) & 20 \% & 40 \% & 0.1756 \\ - \text { oral 5-ASA (\%) } & 93.3 \% & 94.3 \% & 0.8976 \\ - \text { rectal 5-ASA } & 60 \% & 51.4 \% & 0.5812 \\ (\%) & & 0.6678 \\ - \text { immune } & 20 \% & 25.7 \% & \\ \text { modulator (\%) } & & & 0.1266 \\ -\quad \text { biologic } & 6.7 \% & 0 \% & \\ \text { agent (\%) } & & & \end{array}$

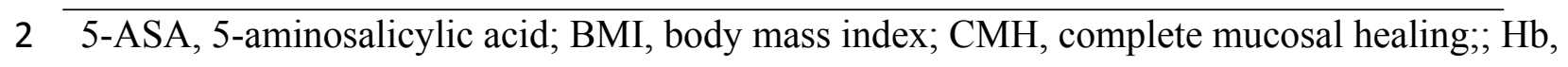

3 hemoglobin; iFOBT, immune fecal occult blood test; WBC, white blood cell 


\section{Table 3 (on next page)}

Comparison of patients with and without endoscopic mucosal healing 
1 Table 3. Comparison of patients with and without endoscopic mucosal healing

\begin{tabular}{|c|c|c|c|}
\hline & EMH $(+)(n=31)$ & EMH $(-)(n=19)$ & $P$-value \\
\hline Age (mean, SD) & $47.16(15.26)$ & $45.26(9.38)$ & 0.628 \\
\hline WBC count & $6.1(5.1-7.7)$ & $7.4(5.5-8.7)$ & 0.2630 \\
\hline \multicolumn{4}{|l|}{ (median, IQR) } \\
\hline $\mathrm{Hb}$ (median, IQR) & $14.2(13.4-15.1)$ & $12.7(9.9-14.7)$ & 0.0324 \\
\hline Platelet (median, & $246(207-281)$ & $319(249-403)$ & 0.0115 \\
\hline \multicolumn{4}{|l|}{ IQR) } \\
\hline iFOBT, ng/mL & $7(7-29.5)$ & $283(129.3-400)$ & $<0.0001$ \\
\hline \multicolumn{4}{|l|}{ (median, IQR) } \\
\hline $\mathrm{FC}, \mu \mathrm{g} / \mathrm{g}$ (median, & $71.2(39.1-222.8)$ & $912(448.8-1800)$ & 0.0002 \\
\hline \multicolumn{4}{|l|}{ IQR) } \\
\hline Disease & $8 / 14 / 9$ & $5 / 6 / 8$ & 0.5665 \\
\hline \multicolumn{4}{|l|}{ distribution } \\
\hline \multicolumn{4}{|l|}{ (E1/E2/E3) } \\
\hline Mayo score stool & $11 / 16 / 3 / 1$ & $0 / 6 / 9 / 4$ & 0.0001 \\
\hline \multicolumn{4}{|l|}{ frequency $(0 / 1 / 2 / 3)$} \\
\hline Mayo score rectal & $23 / 6 / 2 / 0$ & $6 / 5 / 6 / 2$ & 0.0079 \\
\hline \multicolumn{4}{|l|}{ bleeding $(0 / 1 / 2 / 3)$} \\
\hline Physician rating of & $15 / 15 / 1 / 0$ & $1 / 11 / 6 / 1$ & 0.0015 \\
\hline \multicolumn{4}{|l|}{ disease activity } \\
\hline$(0 / 1 / 2 / 3)$ & & & \\
\hline
\end{tabular}

Current medication 


$\begin{array}{llcl}- \text { steroids (\%) } & 22.6 \% & 52.6 \% & 0.0311 \\ - \text { oral 5-ASA (\%) } & 93.55 & 94.7 \% & 0.8650 \\ - \text { rectal 5-ASA } & 54.8 \% & 52.6 \% & 0.8804 \\ (\%) & & 0.3308 \\ - \text { immune } & 19.4 \% & 31.6 \% & \\ \text { modulator (\%) } & & & 0.4337 \\ -\quad \text { biologic } & 3.2 \% & 0 \% & \\ \text { agent }(\%) & & & \end{array}$

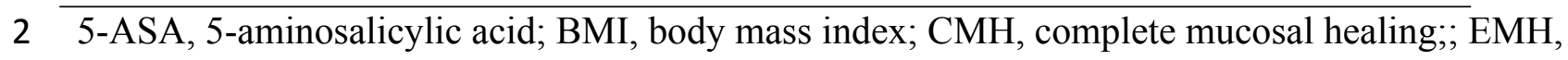
3 endoscopic mucosal healing;; Hb, hemoglobin; iFOBT, immune fecal occult blood test; WBC, 4 white blood cell 
Table 4 (on next page)

Correlations of endoscopy activity with iFOBT, FC, ESR, and CRP 
1 Table 4. Correlations of endoscopy activity with iFOBT, FC, ESR, and CRP

\begin{tabular}{|c|c|c|c|c|c|}
\hline $\begin{array}{c}\text { Mayo } \\
\text { Endoscopy } \\
\text { Score }\end{array}$ & & 0.708 & 0.449 & 0.548 & 0.497 \\
\hline iFOBT & $0.708, \mathrm{p}<0.0001$ & & 0.481 & 0.568 & 0.316 \\
\hline ESR & $0.449, \mathrm{p}=0.0012$ & $\begin{array}{c}0.481, \\
\mathrm{p}=0.0005\end{array}$ & 0.312 & 0.607 \\
\hline FC & $0.548, \mathrm{p}<0.0001$ & $\begin{array}{c}0.568, \\
\mathrm{p}<0.0001\end{array}$ & $\begin{array}{c}0.312, \\
\mathrm{p}=0.0291\end{array}$ & 0.317 \\
\hline CRP & $0.497, \mathrm{p}=0.0003$ & $\begin{array}{c}0.316, \\
\mathrm{p}=0.0271\end{array}$ & $\begin{array}{c}0.607, \\
\mathrm{p}<0.0001\end{array}$ & $\begin{array}{c}0.317, \\
\mathrm{p}=0.0266\end{array}$ \\
\hline & Mayo Endoscopy & iFOBT & ESR & FC & CRP \\
\hline
\end{tabular}

Spearman rank correlation coefficient. CRP, C-reactive protein; ESR, erythrocyte sedimentation rate; $\mathrm{iFOBT}$, immune fecal occult blood test; FC, fecal calprotectin 


\section{Table 5 (on next page)}

Literature comparing the use of FC and iFOBT for the prediction Mayo endoscopic mucosal healing among patients with UC 
1 Table 5. Literature comparing the use of FC and iFOBT for the prediction Mayo endoscopic

2 mucosal healing among patients with UC

\begin{tabular}{|c|c|c|c|}
\hline $\begin{array}{l}\text { Author, } \\
\text { Region }\end{array}$ & $\begin{array}{l}\text { Case } \\
\text { Number, } \\
\text { Study Type, }\end{array}$ & Description of Results & Year \\
\hline Yen, Taiwan & $50, \mathrm{R}, \mathrm{S}$ & $\begin{array}{l}\text { No difference in AUC of FC (cutoff level, } \\
156 \mu \mathrm{g} / \mathrm{g} \text { ) vs. iFOBT (cutoff level, } 30 \mathrm{ng} / \mathrm{mL} \text { ) } \\
\text { for } \mathrm{MES}=0 \text { vs. MES } \geq 1(0.769 \text { vs. } 0.813, \mathrm{P}= \\
0.5581 \text { ) } \\
\text { No difference in AUC of FC (cutoff level, } \\
156 \mu \mathrm{g} / \mathrm{g} \text { ) vs. iFOBT (cutoff level, } 43 \mathrm{ng} / \mathrm{mL} \text { ) } \\
\text { for } \mathrm{MES} \leq 1 \text { vs. } \mathrm{MES} \geq 2(0.812 \text { vs. } 0.906, \mathrm{P}= \\
0.1207)\end{array}$ & $\begin{array}{l}\text { Present } \\
\text { Study }\end{array}$ \\
\hline $\begin{array}{l}\text { Naganuma, } \\
\text { Japan }\end{array}$ & $429, \mathrm{P}, \mathrm{M}$ & $\begin{array}{l}\text { No difference in AUC of FC (cutoff level, } 146.0 \\
\mathrm{mg} / \mathrm{kg} \text { ) vs. iFOBT (cutoff level, } 77.0 \mathrm{ng} / \mathrm{mL} \text { ) for } \\
\mathrm{MES}=0 \text { vs. MES } \geq 1(0.7774 \text { vs. } 0.8085, \mathrm{P}= \\
0.394) \\
\text { No difference in AUC of FC (cutoff level, } 277.0 \\
\mathrm{mg} / \mathrm{kg} \text { ) vs. iFOBT (cutoff level, } 201.0 \mathrm{ng} / \mathrm{mL}) \\
\text { for MES } \leq 1 \text { vs. MES } \geq 2(0.8166 \mathrm{vs.} 0.8353, \mathrm{P}= \\
0.394)\end{array}$ & $2020[17]$ \\
\hline
\end{tabular}




\begin{tabular}{|c|c|c|c|}
\hline Kim, Korea & $127, \mathrm{P}, \mathrm{M}$ & $\begin{array}{l}\text { AUC of FC (cutoff level, } 70 \mu \mathrm{g} / \mathrm{g} \text { ) }>\mathrm{iFOBT} \\
\text { (cutoff level, } 0 \mathrm{ng} / \mathrm{mL} \text { ) for } \mathrm{MES}=0 \mathrm{vs} . \mathrm{MES} \geq \\
1(0.858 \text { vs. } 0.707, \mathrm{P}=0.0009) \\
\text { No difference in AUC of } \mathrm{FC} \text { (cutoff level, } 200 \\
\mu \mathrm{g} / \mathrm{g}) \text { vs. iFOBT (cutoff level, } 60 \mathrm{ng} / \mathrm{mL} \text { ) for } \\
\mathrm{MES} \leq 1 \text { vs. } \mathrm{MES} \geq 2(0.82 \text { vs. } 0.813, \mathrm{P}=0.089)\end{array}$ & $2020[16]$ \\
\hline Ryu, Korea & $128, \mathrm{P}, \mathrm{S}$ & $\begin{array}{l}\text { AUC of FC (cutoff level, } 170 \mu \mathrm{g} / \mathrm{g} \text { ) }>\text { iFOBT } \\
\text { (cut-off value, } 100 \mathrm{ng} / \mathrm{mL} \text { )for } \mathrm{MES}=0 \mathrm{vs.} \mathrm{MES} \\
\geq 1(0.847 \text { vs. } 0.757, \mathrm{P}<0.0001) \\
\text { AUC of FC (cutoff level, } 170 \mu \mathrm{g} / \mathrm{g})>\text { IFOBT } \\
\text { (cut-off value, } 100 \mathrm{ng} / \mathrm{mL} \text { ) for } \mathrm{MES} \leq 1 \mathrm{vs} \text {. } \\
\text { MES } \geq 2(0.863 \text { vs. } 0.765, \mathrm{P}<0.0001)\end{array}$ & $2019[18]$ \\
\hline Kim, Korea & $68, \mathrm{R}, \mathrm{S}$ & $\begin{array}{l}\text { No difference in AUC of FC vs. iFOBT for } \\
\mathrm{MES} \leq 1 \text { vs. } \mathrm{MES} \geq 2(0.727 \text { vs. } 0.717, \mathrm{P}=\end{array}$ & $2018[20]$ \\
\hline $\begin{array}{l}\text { Takashima, } \\
\text { Japan }\end{array}$ & 92,P,S & $\begin{array}{l}\text { No difference in AUC of FC (cut-off value, } 200 \\
\mu \mathrm{g} / \mathrm{g} \text { ) vs. } \mathrm{iFOBT} \text { (cut-off value, } 75 \mathrm{ng} / \mathrm{ml} \text { ) for } \\
\mathrm{MES}=0 \text { vs. } \mathrm{MES} \geq 1(0.83 \text { vs. } 0.82, \mathrm{P}=0.394) \\
\text { No difference in AUC of FC (cut-off value, } 369 \\
\mu \mathrm{g} / \mathrm{g}) \text { vs. iFOBT (cut-off value, } 280 \mathrm{ng} / \mathrm{ml}) \text { for } \\
\mathrm{MES} \leq 1 \text { vs. } \mathrm{MES} \geq 2(0.80 \text { vs. } 0.79, \mathrm{P}=0.394)\end{array}$ & $2015[19]$ \\
\hline
\end{tabular}


P: prospective; S: single-center study; M: multicenter study 
Figure 1

Figure 1 Correlation of complete Mayo score with iFOBT (A, Left) and FC (B, Right) (A, Left) Spearman's coefficient of rank correlation $=0.737, \mathrm{P}<0.0001$ (B, Right) Correlation of FC with a complete Mayo Score
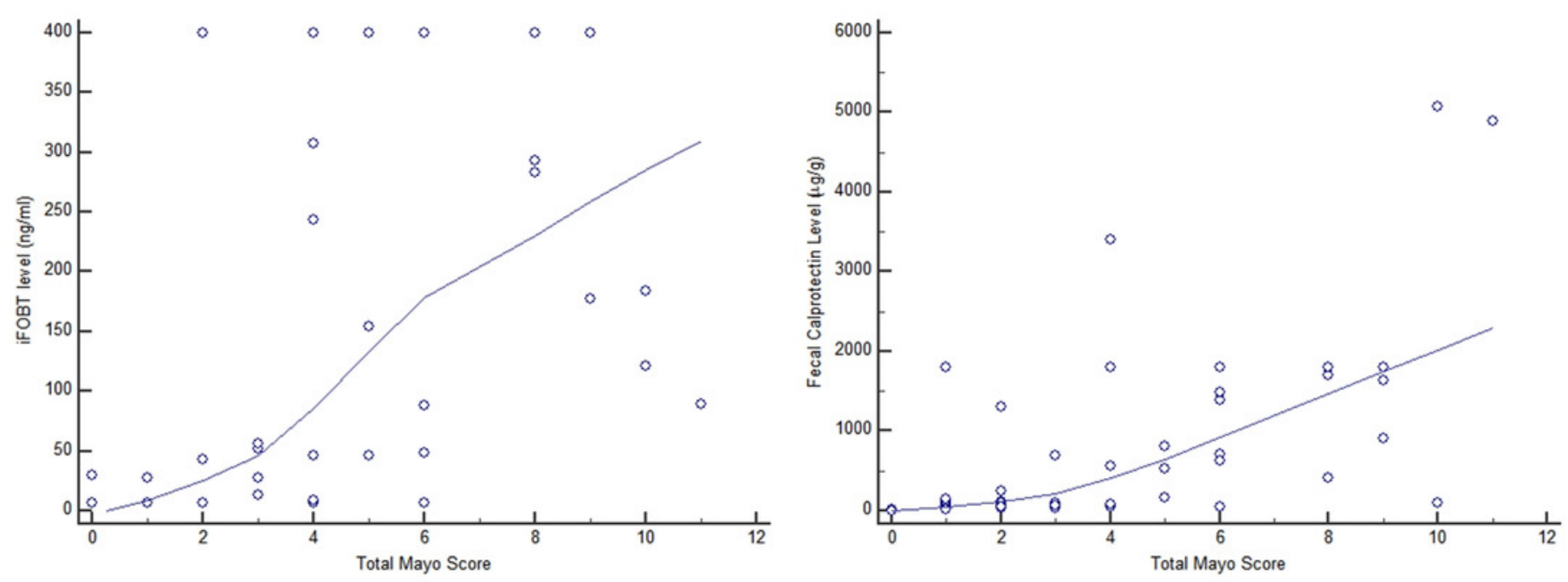


\section{Figure 2}

Figure 2. ROC curve analysis for the use of iFOBT to assess complete mucosal healing (A, Left) and endoscopic mucosal healing (B, Right).

(A, Left ) Using an iFOBT criterion of $\leq 30 \mathrm{ng} / \mathrm{mL}(95 \% \mathrm{Cl} \leq 27$ to $\leq 52)$, the sensitivity is $93.33 \%$, and the specificity is $71.43 \%$ for predicting complete mucosal healing. Dot line: $95 \%$ Confidence interval of ROC curve. (B, Right) Using an iFOBT criterion of $\leq 43 \mathrm{ng} / \mathrm{mL}(95 \% \mathrm{Cl}$ $\leq 28$ to $\leq 88$ ), the sensitivity is $80.65 \%$, and the specificity is $100 \%$ for predicting endoscopic mucosal healing. Dot line: $95 \%$ Confidence interval of ROC curve.
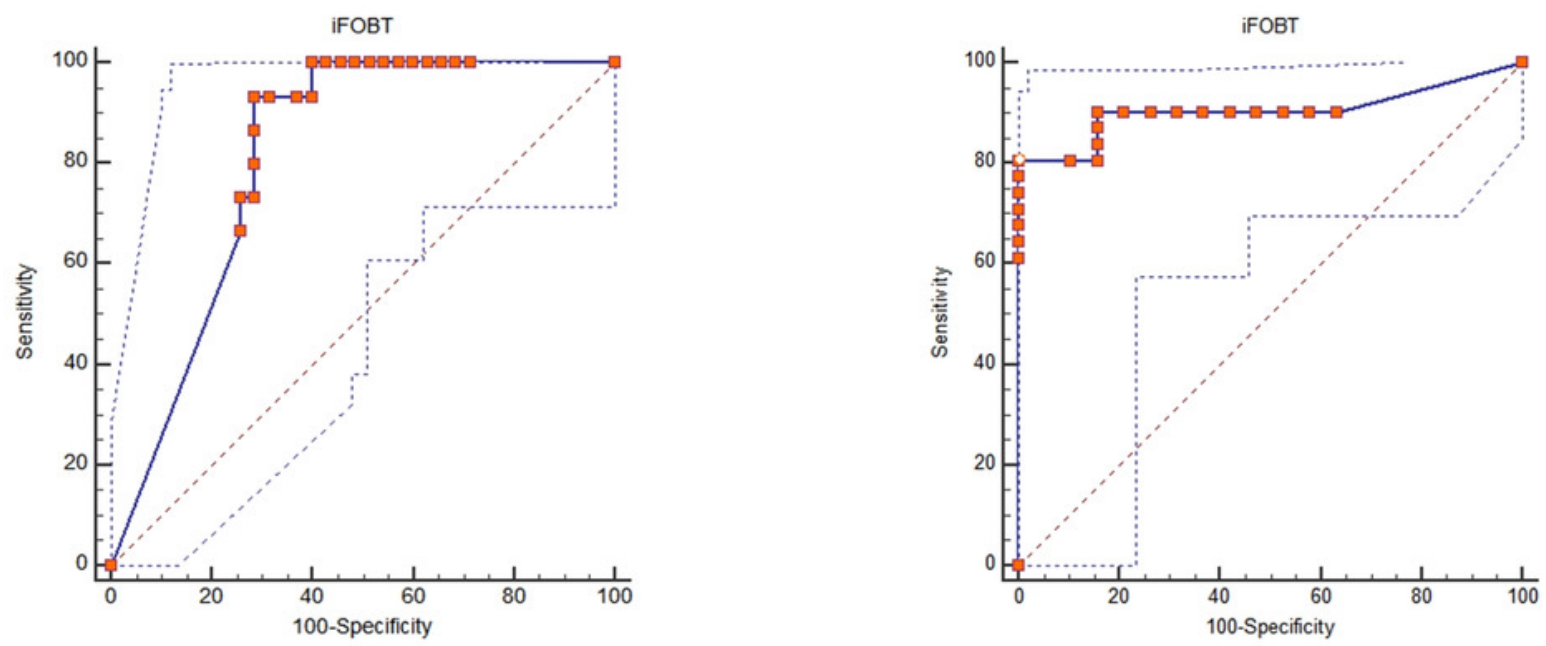
Figure 3

Figure 3. ROC curve analysis for the use of FC to assess complete mucosal healing ( $A$, Left) and endoscopic mucosal healing (B, Right).

(A, Left) Using an FC criterion of $\leq 156 \mu \mathrm{g} / \mathrm{g}(95 \% \mathrm{Cl} \leq 100$ to $\leq 1800)$, the sensitivity is $86.67 \%$, and the specificity is $62.86 \%$ for predicting complete mucosal healing. Dot line: $95 \%$ Confidence interval of ROC curve. (B, Right) Using an FC criterion of $\leq 156 \mu \mathrm{g} / \mathrm{g}(95 \% \mathrm{Cl}$ $\leq 63.7$ to $\leq 638.9$ ), the sensitivity is $74.19 \%$ and the specificity is $84.21 \%$ for predicting endoscopic mucosal healing.Dot line: 95\% Confidence interval of ROC curve.
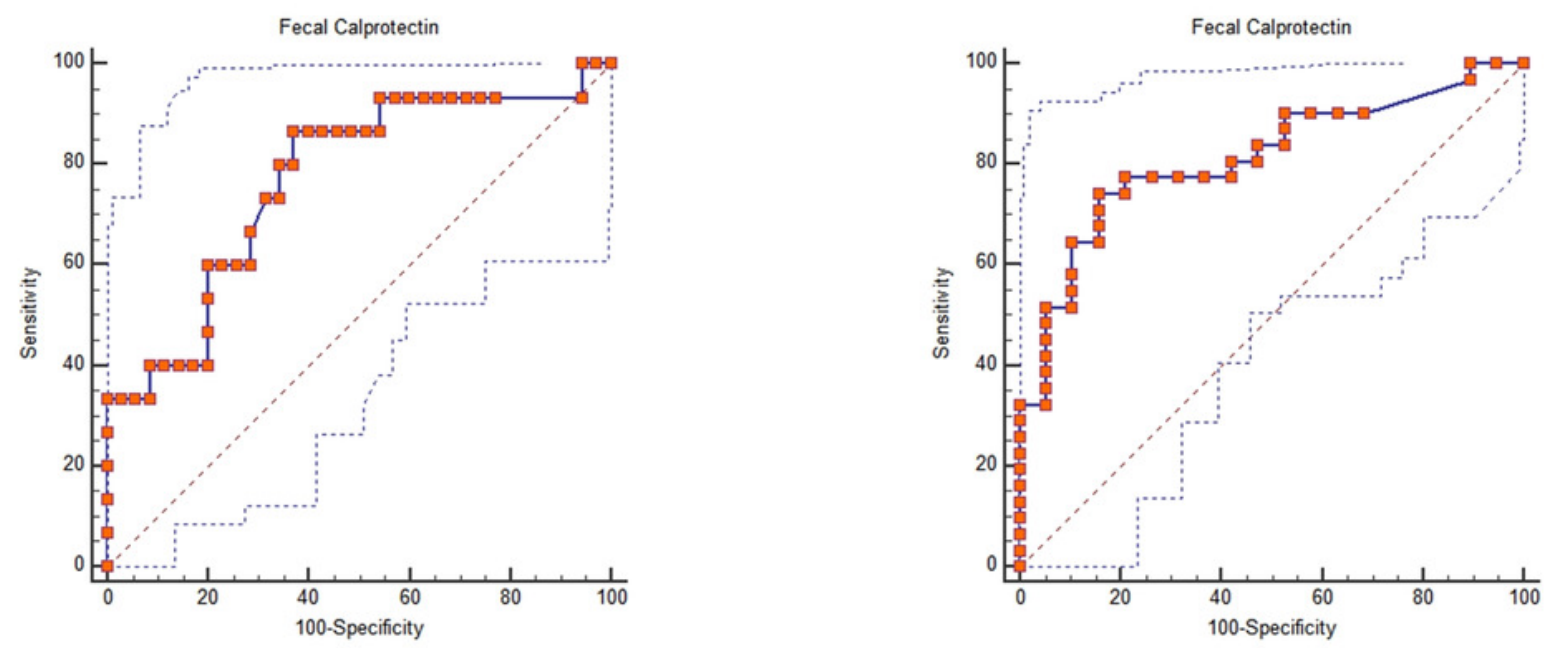
Figure 4

Figure 4. ROC curve comparing iFOBT and FC for predicting complete mucosal healing (A, Left) and endoscopic mucosal healing (B, Right).

(A, Left) Pairwise comparison of ROC curves: iFOBT vs. FC, $p=0.5581$. (B, Right) Pairwise comparison of ROC curves: iFOBT vs. FC, $p=0.1207$.
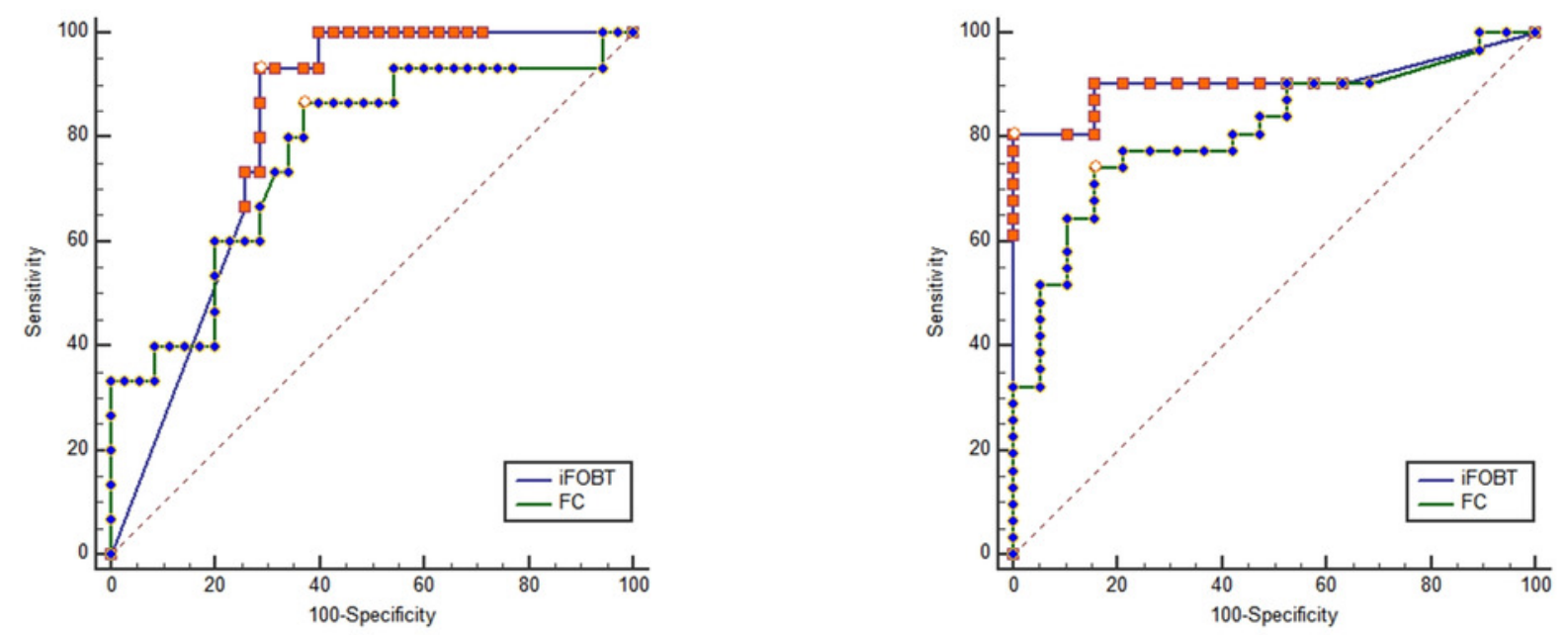